\title{
Is the Unavailability of Partograph a Reason for its Low Usage? The Case of Jimma University Medical Center
}

\author{
Belina $\mathrm{S}^{1 *}$, Negro $\mathrm{B}^{2}$, Getachew $\mathrm{M}^{3}$, Alemu $\mathrm{T}^{4}$ and Girma $\mathrm{E}^{5}$ \\ ${ }^{1}$ Department of Nursing and Midwifery, University of Jimma, Ethiopia \\ ${ }^{2}$ Department of Population and Family Health, University of Mettu, Ethiopia \\ ${ }^{3}$ Department of Obstetrics and Gynecology, University of Wollega, Ethiopia \\ ${ }^{4}$ Department of Population and Family Health, University of Jimma, Ethiopia \\ ${ }^{5}$ Department of Preventive Medicine, University of Addis Ababa, Ethiopia
}

\begin{abstract}
Background: Partograph is a pre-printed paper that provides a visual display of recorded observations carried out on mother and fetus during labor process. It enables health professionals to monitor feto-maternal wellbeing and progress of labor. It also alerts the care providers to take timely intervention when required. Despite its importance and WHO recommendation its utilization is inadequate in low and middle income countries. Therefore, the aim of this study is to explore barriers for utilization of partograph during labor delivery at Jimma University Medical Center 2017.

Methods: A programmatic research design was employed. Data was collected through key informant interview and client record review. A purposive sampling technique was used to select the key informants and random sampling was used to select the client's document. Data were collected using interview guide and check list for record review.

Results: Data were analyzed qualitatively by means of the ATLAS.ti 7 software and four themes emerged namely, type of the institution, perception of care providers to ward partograph, previous trends, and absence of follow up and control mechanism. The study found that some health workers were more informed about the importance of partograph, others have negative attitude on its utilization. The study also revealed the use of partograph was determined by diverse factors like type of the institution, perception of care providers toward partograph, previous situation (trends), lack of controlling mechanism. Therefore, availing monitoring mechanisms and periodic review of trends of utilization reviewing trends, securing sufficient staffing, and training to brining all care providers under same umbrella as some of the health workers had little knowledge which was evidenced by record review and negative attitude are recommended.
\end{abstract}

Keywords: Partograph; Barriers; Jimma University; Medical center

Acronyms: BP: Blood Pressure; JUMC: Jimma University Medical Center; JUSH: Jimma University Specialized Hospital; KII: Key Informants Interview; U/A: Urine Analysis; WHO: World Health Organization.

\section{Introduction}

A partograph is a pre-printed paper that provides a visual display of recorded observations carried out on feto-maternal condition during labor process. It is universally used as part of Safe Motherhood Initiative for improving labor management and reducing maternal and fetal morbidity and mortality $[1,2]$. It gained popularity in the 1970's and today most labor and delivery wards use it. A large and growing body of literature shows that its correct use lowers rates of prolonged labors and associated complications, fewer surgical interventions. Electronic partograph are becoming more widespread and can be built into medical records systems [3]. The World Health Organization (WHO) promotes the use of the partograph to monitor and improve the management of labor and to support decision-making regarding interventions According to the study conducted among health professionals in Amhara region, Jimma University Specialized Hospital, 99\% participants mentioned at least one component of it but only 77 (26.6\%) participants' had mean score or more on the knowledge questions used to measure knowledge of components of the partograph. Two hundred sixty participants (90\%) reported that utilization of the partograph would help making timely decision during labor, also the study revealed that among the total 160 charts reviewed, although $128(80 \%)$ of them were having the partograph paper attached, only $58(45.3 \%)$ charts were filled, moreover among those filled, only 17 (29.3\%) of them were to the standard and out of 340 a reviewed maternal records Jimma University Specialized Hospital, 274 (80.6\%) had partograph but it was used for 19 (6.9\%) mothers and the momentous predictors were: time of admission, nature of membrane on admission $[4,5]$.

The study conducted at Addis Ababa among obstetric care givers in public health institutions showed that the reasons for not using the partograph were lack of awareness, knowledge deficits, time consuming, staff in adequacy and lack of training [6]. The study conducted in Ghana to ascertain the proportion and correct use of the partograph reveals that half of the labor cases were not monitored with it and in the other half the progress of labor parameters, fetal wellbeing and maternal wellbeing were not monitored to the standard. The reasons for nonutilization were reported as lack of charting skills, failure to appreciate it's use in monitoring the progress of labor [7]. The study conducted in the Niger Delta Region of Nigeria, Uganda to assess the utilization of partograph charts revealed that reasons for not using it were lack of adequate partograph, shortage of staff, little or knowledge in the use of the partograph, inadequacy of sphygmomanometer, fetoscopes, lack

*Corresponding author: Sena Belina, Department of Nursing and Midwifery, University of Jimma, Ethiopia, Tel: 251912 112 666; E-mail: senabalina26@gmail.com

Received April 24, 2018; Accepted August 28, 2018; Published September 05, 2018

Citation: Belina S, Negro B, Getachew M, Alemu T, Girma E (2018) Is the Unavailability of Partograph a Reason for its Low Usage? The Case of Jimma University Medical Center. Health Care Current Reviews 6: 225. doi: 10.4172/23754273.1000225

Copyright: ( $) 2018$ Belina S, et al. This is an open-access article distributed unde the terms of the Creative Commons Attribution License, which permits unrestricted use, distribution, and reproduction in any medium, provided the original author and source are credited. 
of ongoing support supervision, rotation of staff after every 3 months and transferring of staffs to other hospitals, heavy workload by staffs brought about by inadequate numbers of human resources and the presence of two types of partograph and it was time consuming [8-10].

Despite the WHO recommendation of universal use of the partograph. About its utilization at Jimma University Medical Center is not well known. Therefore, this study is designed to explore the reasons for not utilizing partograph in the area hoping that the answers to this question will be important to inform policies and strategies in the provision of maternity care services in the country, especially in the areas of preventing prolonged labor and its attendant complications and it can be used as baseline evidence same inquiries.

\section{Method and Materials}

The study was conducted in June, 2017 at Jimma University Medical Center which is one of the oldest public hospitals established in 1937 as Jimma hospital, later Jimma University Specialized Hospital (JUSH) which is the only teaching and referral hospital in the South Western part of the country. The hospital has different departments those renders comprehensive health services by professionals of different qualification level and disciplines [11]. Descriptive qualitative study design was employed. Data was collected from Midwives, Chief residents who have been rendering the service at maternity unit at for the last two years based on their position, nearness to the activity, experience were purposively selected as key informants and eight randomly selected mothers' chart who have been delivered at this hospital (JUMC) to determine utilization and completeness of the partograph. Proceeding to data collection discussions were held with Nursing Service Director and supervisory team leader of the maternity ward to identify individuals who met part or all of the established condition. Accordingly, four individuals that met the criterion were nominated. Data was collected using key informants' interview (KII) guide and observation check list for the document review. The taperecorded interviews were transcribed to local language and were later translated into English.

Our data consisted largely of interview transcripts, document review and expanded field notes. Four members of the research team independently coded the transcripts following the objectives and emerging themes from the data using ATLAS.ti software. Accordingly, findings were categorized in to different themes and sub-themes. Type of the institution, perception of care providers toward the partograph, previous trends, absence of follow up were identified as outstanding themes. Elucidations of the results follow the respective themes and verbatim that capture dominant views were considered wherever appropriate to substantiate the findings. Data collectors were the research teams themselves who have familiarity with the local culture, fluent speaker of the local languages and experience on qualitative research method. Ethical clearance was sought and obtained from Jimma University, Department of Population and Family Health. Participation was entirely voluntary and participants were informed that at any time during the interview, they could opt out. Participants received an explanation about the purpose of the study, invited to participate and asked to provide oral consent. De-identification and confidentiality were ensured by using numbers and fictitious names to describe and identify participants.

\section{Results}

The data were collected through review of randomly selected client charts and purposely selected key informants. The age of the participants ranged from 26 to 32 years with experience between 4 and 7 years. All participants betrothed well with the topic and responded enthusiastically to the questions. The findings are presented in four thematic areas namely, type of the institution/Health facility, perception of care providers to ward partograph, previous situation /trends, absence of follow up and controlling mechanism All the reviewed charts, had partograph attached but documentation in all of them were below standard. The findings by sub-group have therefore been incorporated within each theme, and presented as a whole.

\section{Type of the institution}

All of the key informants had consensus on partograph utilization and they painted their view as its implication to detect labor abnormality is not questionable. There was a general agreement on its increased use at JUMC when compared with the last three-four years. Also most of the participants described its utilization is not customarily because Jimma University Medical Center is only teaching and referral hospital in South West part of the country where number of students from diverse disciplines are practicing and rotating once in a while, cases with sundry obstetrics complications referred from the close to health facilities which makes the caseloads of the hospital demanding and lack of clear job distribution among the medical interns, Residents, other medical students and staff midwifes/Nurses are among barriers for the low utilization of it. However, they rated it regarding its potential on reducing maternal, perinatal morbidity and mortality as well its effect quality of care, and recommended its utilization. For instance, a year four obstetrics and gynecology resident stated that:

... This Hospital is a teaching institution the students are rotating once in a while, you teach, show the first team, after sometime the will rotate and other team will comes tomorrow again you teach, show them they will rotate, in addition there are case load, at night time there is time when you move to other case before completing the others history due to case load, the time may not enough to go to partograph after completing the history of the client/s as we are not only manage Labor by partograph only and some care providers perceive as partograph is used only by care providers at low level to identify abnormalities (Year IV obstetrics and gynecology resident).

\section{Care providers' attitude toward partograph}

Care providers 'perception about the importance of partograph is one of the concerns redundantly pointed out by the participants. The participants undermined the significance of partograph in obstetric care and believed it to be more apt for lower level care providers. For example one of the key informants expressed her experience: It is presented in simplest way to detect abnormality in labor early, enables to make a decision on steps of managements and for handover the cases when going out off duty for the next care providers, as to the availability it present adequately, to say the skill gap it presented in simplest way, even if there is skill gap it can be resolved through communication within few days because mixed professionals with different levels of qualifications are here. Therefore, for me the main thinning is perception of care providers about partograph, for the reason that in majority of client charts there is chart contains selected points on partograph like fetal heartbeat, maternal vital sign ...thus posted on wall and used as partograph what we call labor follow up chart instead of partograph, actually this labor follow up chart is used during latent phase but there are care providers use this labor follow up chart throughout the labor process believing it is equivalent to partograph and partograph is time consuming. 


\section{Care providers' attitude toward partograph}

Care providers 'perception about the importance of partograph is one of the concerns redundantly pointed out by the participants. The participants undermined the significance of partograph in obstetric care and believed it to be more apt for lower level care providers. For example one of the key informants expressed her experience:... it is presented in simplest way to detect abnormality in labor early, enables to make a decision on steps of managements and for handover the cases when going out off duty for the next care providers, as to the availability it present adequately, to say the skill gap it presented in simplest way, even if there is skill gap it can be resolved through communication within few days because mixed professionals with different levels of qualifications are here. Therefore, for me the main thinning is perception of care providers about partograph, for the reason that in majority of client charts there is chart contains selected points on partograph like fetal heartbeat, maternal vital sign ...thus posted on wall and used as partograph what we call labor follow up chart instead of partograph, actually this labor follow up chart is used during latent phase but there are care providers use this labor follow up chart throughout the labor process believing it is equivalent to partograph and partograph is time consuming. (Age 28, had six years' experience, Midwife)

\section{Previous situation /trends}

The partograph is a very useful graphical record of the course of labor that yields optimum results when employed in labor management by obstetric caregivers. It is what strongly recommended by World Health Organization as its proper application will culminate in a remarkable reduction in the incidence and outcomes of prolonged and obstructed labor, which are reported to be associated with $8 \%-10 \%$ of maternal deaths. The participants also reflected their concern on its importance but there is trend in this institution which are inherited from the seniors.

One of the participant explained this saying: The main thinning is that the trends from the previous staffs if you come to this ward before three years no body care about the partograph. ... still I don't remember the complication occurred due to not utilizing the partograph this may be due to presence of senior staffs those can detect the occurrences of it ahead instead of alert and action line also not occurred for time being does not mean it would not occur in future so better if we use it at all time, As this is teaching institution those graduate from here and deployed every part of the country if fail to understand it well, not interprets it well may face challenge as you are not always with them.. (Age 29, had five years' experience, Midwife).

\section{Absence of follow up and controlling mechanism}

The participants also signposted as the use of the partograph is hindered by diverse factors beyond the types of institution and care providers, a notable fact is that the absence of follow up controlling system from different stack holders like minster of health. In our case lack of follow up found as major obstacles in its utilization. For instance, one of the participants expressed his experience saying

"We have copies of partograph; its utilization is very limited because of poor controlling system for its routine use and the care providers do not want to plot it throughout the labor. In fact, we have no guideline but the partographis clear and anybody can use it easily. I am here for the last three years but I didn't see anybody who come to here and evaluated utilization of it from the ministry as well as the hospital managerial body. What I know as good thinning is that our seniors raise this issue on morning and they strongly recommend its utilization, if it continuous like this the possibility of utilizing it becomes increase ...but what we discuss on morning is abnormal labor not utilizing it for non-normal is nothing and no way to evaluated whether it used for normal labor or not by seniors is less likely. Therefore, this is the responsibility of resident and interns, even if it used for all normal labor it increases it utilization". (Year IV obstetrics and gynecology resident).

\section{Discussion}

Despite the recommendation of WHO for universal utilization of partograph as part of Safe Motherhood Initiative for improving labor management and reducing maternal and fetal morbidity and mortality but study revealed that its utilization was limited [2]. If operated properly it will contribute in a remarkable reduction in the incidence and outcomes of prolonged and obstructed labor, which are reported to be associated with $8 \%-10 \%$ of maternal deaths [5]. In this study the partograph is present attached to all of the reviewed mothers document/charts. However, in none of the reviewed documents it was piloted to the standard, in some cases the way piloting started was not consistent with the up-to-date recommendation of WHO, the first piloting was not on alert line, in some documents the labor follow up chart is used as a substitute of the partograph on which selected points are noted (Figure 1).

The partakers were also agreed with the importance of its utilization and improvement in its usage when compared with the past threefour years even though it is still not customarily used because of the characteristics of the hospital which is the only teaching and referral hospital in South West part of the country where number of students from diverse disciplines are practicing and rotating once in a while, cases with sundry obstetrics complications referred from the close to health facilities which makes the case loads of the hospital demanding and upshot in less the utilization of the it. This finding is consistent with the study conducted among health professionals in Amhara region that indicated as more than half of the care providers had good knowledge of partograph and the format was presented attached to the client charts but only in less than one third it was properly filled to monitor the progress of labor [4].

Also it is compliant with the study done in Uganda that shown the reasons for not utilizing partograph were staff rotation every three months and transfer to other hospitals, heavy workload brought about by insufficient numbers of human resources, the study conducted Calabar Teaching Hospital, Nigeria where only 2 workers were allocated on shift at each time, overwhelming delivery rate and this shortage of staff are responsible for non-utilization of the partograph and the

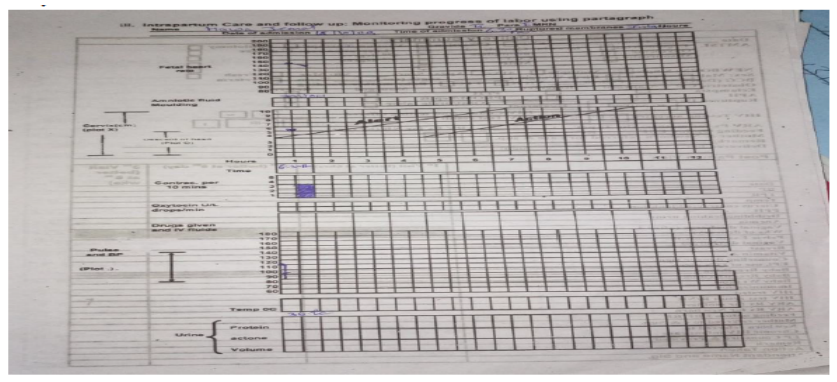

Figure 1: Partograph utilized at maternity, Jimma University Medical Cente that indicate its incompleteness and the first pilot is not on alert line, Jimma University Medical center. 
Citation: Belina S, Negro B, Getachew M, Alemu T, Girma E (2018) Is the Unavailability of Partograph a Reason for its Low Usage? The Case of Jimma University Medical Center. Health Care Current Reviews 6: 225. doi: 10.4172/2375-4273.1000225

study conducted at Jimma University Specialized Hospital were the utilization of partograph was very low as only 19/274 (6.9\%) of mothers delivered at the hospital were monitored with it, while $274(80.6 \%)$ mothers document had a partograph in their file. Of those used only in two cases the records of the mothers' monitored with it (Partograph) had correct documentation of some of the key events which indicates poor monitoring of the key events against standards [5,9,10]. This study also acknowledged as care providers' perception about the worth of partograph is among the points redundantly pointed out by the participants. Some of the participants were reported that some of the care providers believe as it is not significant, time consuming and it is tool for low level care provider. Some were used locally prepared labor follow up chart rather than the partograph seeing the partograph is time consuming tool (Figure 2).

This result is consistent with findings of the study conducted in Nigeria that revealed one of the reasons for not using it was believing piloting of partograph is time consuming and the study conducted at Jimma University Specialized Hospital were the barriers for its nonutilization were staff shortage, lack of protocol, attitude of care providers, time of admission of mothers for delivery, lack of controlling system and availability of other modern tools $[5,8]$. The study participants likewise signposted as the use of the partograph is hindered by diverse factors beyond the types of institution and care providers, a notable fact is that the absence of follow up controlling system from different stack holders like mister of health. In our case lack of follow up found as major obstacles in its utilization. For instance, one of the participants expressed his experience saying:

"We have copies of partograph; its utilization is very limited because of poor controlling system for its routine usage. In fact, we have no guideline but the partograph is clear and anybody can use it easily. I am here for the last three years but I didn't see anybody who come to here and evaluated utilization of it from the ministry as well as the hospital managerial body. What I know as good thinning is that our seniors raise this issue on morning and they strongly recommend its utilization if it continuous like this the possibility of utilizing it becomes increase ...but what we discuss on morning is the abnormal labor not utilizing it for non-normal is nothing ... and no way to evaluated whether it used for normal labor or not by them is less likely". This finding is in line with the study indicated in Uganda that revealed the reasons for not utilizing partograph were lack of ongoing support supervision and clinical audits, the study conducted at Jimma University Specialized Hospital where lack of protocol, lack of controlling system were among the barriers for its $[5,10]$.

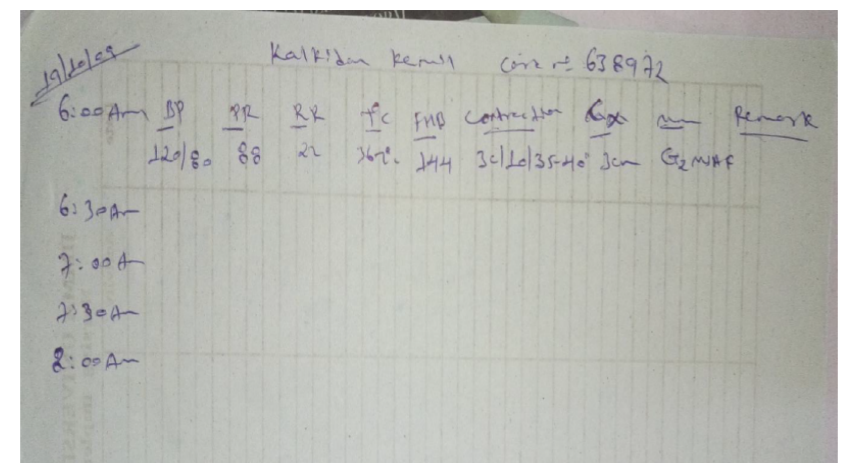

Figure 2: Locally prepared labor follow up chart used at maternity, Jimma University Medical Center, Jimma University Medical center.

\section{Conclusions}

This study highlighted as some health workers were more informed about the importance of partograph while others had little knowledge on the modified partograph as it evidenced by the document review. Also there are staffs who have negative attitude on partograph use and documentation still persisted and this could have effect on its utilization. In general, this study identified that utilization of partograph were determine by diverse factors like type of the institution, perception of care providers, previous situation(trends), absence of follow up. This requires fundamental reform and introduction of comprehensive interventions at different levels to increase it utilization

\section{Recommendation}

Based on the findings of the study the following recommendations were forward

- Availing constant monitoring, supervision and review of the utilization by health workers and trends of the its usage should communicated to the staffs so that they may develop a sense of ownership for the utilization.

- Making sufficient staffing also crucial to offer and achieve quality partograph use.

- The training was conducted for thus not enabling to demonstration as some health workers had little knowledge on the partograph and negative attitude on partograph use.

\section{Acknowledgement}

We would like to express our deepest gratitude to Jimma University Institute of health Department of Population and Family Health and study participants for their backing and providing valuable information in this study and professor Susan Anand for language edition.

\section{References}

1. WHO (2014) WHO recommendations for augmentation of labour.

2. World Health Organization (2011) Prevention and treatment of pre-eclampsia and eclampsia.

3. O'Driscoll K, Gallagher JT (1969) For the prevention of prolonged labor. $\mathrm{Br}$ Med 24: 477-480.

4. Maodo N (2013) Epidemiology and Clinical Study of Pediatric Psoriasis on Black Skin in Dakar, Senegal. Sci J Clin Med 2: 43

5. Kitila S, Gmariam A, Molla A, Nemera (2014) Utilization of partograph and documentation of key parameters. J Preg Child Heal 1: 1-6.

6. Yisma E, Dessalegn B, Astatkie A, Fesseha N (2013) Knowledge and utilization of partograph among obstetric care givers in public health institutions of Addis Ababa, Ethiopia. BMC Pregnancy Childbirth 13: 17.

7. Opoku BK, Nguah SB (2015) Utilization of the modified WHO partograph in assessing the progress of labour in a metropolitan area in Ghana. Res $J$ Women's Heal 2: 2 .

8. Opiah MM, Ofi AB, Essien EJ, Monjok E (2012) Knowledge and utilization of the partograph among midwives in the Niger Delta Region of Nigeria. Afr $J$ Reprod Heal 16: 125-132.

9. Agan T, Akpan U, Okokon I, Oku A Asibong U et al (2014) Assessment of the Knowledge and Utilization of the Partograph among Non-physician Obstetric Care Givers in the University of Calabar Teaching Hospital, Calabar, Nigeria. Br J Med Med Res 4: 5741-5755.

10. Aliona Masika M, Peter Katongole S, Govule P (2015) Improving Partograph Documentation and Use by Health Workers of Bwera Hospital. A Process Improvement Research. Int J Nurs Heal Sci 2: 37-45

11. Dejene S, Negash A, Tesfay K, Jobst A, Abera M (2014) Depression and Diabetes in Jimma University Specialized Hospital, Southwest Ethiopiaf. J Psychiatry 17: 126. 